Draft Version OCtober 29, 2018

Preprint typeset using $\mathrm{L}^{\mathrm{A}} \mathrm{T} \mathrm{E}$ X style emulateapj v. 04/20/08

\title{
OBSERVATIONS OF X-RAY OSCILLATIONS IN $\xi$ BOO: EVIDENCE OF A FAST-KINK MODE IN THE STELLAR LOOPS
}

\author{
J. C. Pandey and A. K. Srivastava \\ Aryabhatta Research Institute of Observational Sciences, Naini tal-263 129, India \\ Draft version October 29, 2018
}

\begin{abstract}
We report the observations of X-ray oscillations during the flare in a cool active star $\xi$ Boo for the first time. $\xi$ Boo was observed by EPIC/MOS of XMM-Newton satellite. The X-ray light curve is investigated with wavelet and periodogram analyses. Both analyses clearly show oscillations of the period of $\sim 1019 \mathrm{~s}$. We interpret these oscillations as a fundamental fast-kink mode of magnetoacoustic waves.

Subject headings: stars: activity, - stars: coronae, - stars: flare, - MHD, - waves
\end{abstract}

\section{INTRODUCTION}

The magnetohydrodynamic (MHD) waves and oscillations in the solar and probably in the stellar atmospheres are assumed to be generated by coupling of complex magnetic field and plasma. These MHD waves and oscillations are one of the important candidates for heating the solar/stellar coronae and accelerating the supersonic winds. In the Sun, magnetically structured flaring loops, anchored into the photosphere, exhibit various kinds of MHD oscillations (e.g. fast sausage, kink and slow acoustic oscillations). The idea of exploiting such observed oscillations as a diagnostic tool for deducing physical parameters of the coronal plasma was first suggested by Roberts et al. (1984) . Wave and oscillatory activity of the solar corona is now well observed with modern imaging and spectral instruments (e.g. instruments on-board /it SOHO, TRACE, HINODE and NoRH ) in the visible, EUV, X-ray and radio bands, and interpreted in terms of MHD wave theory (Nakariakov \& Verwichte 2005).

Stellar MHD seismology is also a new developing tool to deduce the physical properties of the atmosphere of magnetically active stars, and it is based on the analogy of the solar coronal seismology (Nakariakov 2007). The first stellar X-ray flare oscillations in AT Mic have been reported by Mitra-Kraev et al. (2005) and it was interpreted as a signature of slow magneto-acoustic oscillation. Mathioudakis et al. (2006) have reported the fast sausage oscillations of $\sim 10 \mathrm{~s}$ in the flaring stars EQ Peg B. Mullan et al. (1992) have also found the oscillations on the scale of few minutes and interpreted as a transient oscillations in the stellar loops. In this Letter, we find the observational signature of the fast kink oscillations in the magnetically active star $\xi$ Boo $(\mathrm{V}=4.55 \mathrm{mag})$. In the case of solar corona, such type of modes are important to probe the local plasma conditions (e.g., van Doorsselaere et al. 2008). Therefore, such modes may also be useful to diagnose the stellar coronae. $\xi$ Boo is a nearby $(6.0 \mathrm{pc})$ visual binary, comprising a primary G8 dwarf and a secondary K4 dwarf with an orbital period of $151 \mathrm{yr}$. In terms of the outer atmospheric emission, UV and X-ray observations show that the primary dominates entirely over the secondary (e.g. Laming \& Drake 1999). The observations, data reduction, results and discussion along with the conclusions are given in the forthcoming sections.

\section{OBSERVATIONS AND DATA REDUCTION}

The star $\xi$ Boo was observed by RGS and EPIC-MOS on boards XMM-Newton satellite (Jansen et al. 2001) on 2001 January 19 at 19:25:06 UT and lasted for 59 ks. The detailed method of EPIC-MOS data reduction, and the timing, spectroscopic and flare analysis are given in Pandey \& Singh (2008). Here, we used the 0.3-10.0 keV X-ray data observed from EPIC-MOS1. The X-ray light curve of $\xi$ Boo is shown in Figure 1 where flare regions are represented by F1 and F2, and the quiescent state is represented by Q. The light curve was binned with 40s. In the X-ray light curve of $\xi$ Boo, a period of heightened coronal emission ' $U$ ' was identified after the flare F2, where the average flux was 1.8 times more than the quiescent state. We have chosen the $\mathrm{U}$ region for our wavelet and periodogram analyses because of the stable evolution of the integrated $0.3-10 \mathrm{keV}$ light curve after the flare F2. To study the emission in the $\mathrm{U}$ regions only, we fitted the spectral data using 1-temperature plasma model apec (Smith et al. 2001), with the quiescent emission taken into account by including its bestfit 2-temperature model as a frozen background contribution. The best fit temperature and emission measure during the $\mathrm{U}$ part of the light curve are $8.7 \pm 0.3 \mathrm{MK}$ and $3.8 \pm 0.5 \times 10^{51} \mathrm{~cm}^{-3}$, respectively with reduced $\chi^{2}$ per degree of freedom of 0.99. During the flare F2, the average values of temperature and emission measures were found to be $12.0 \pm 0.6 \mathrm{MK}$ and $3.5 \pm 0.1 \times 10^{51} \mathrm{~cm}^{-3}$, respectively.

Line fluxes and positions were measured using the XSPEC package by fitting simultaneously the RGS1 and RGS2 spectra with a sum of narrow Gaussian emission lines convolved with the response matrices of the RGS instruments. The continuum emission was described using Bremstrahlung models at the temperatures of the plasma components inferred from the analysis of EPIC-MOS1 data. The emission measure derived from the analysis of the EPIC data was used to freeze the continuum normalization. For the present purpose we give the line fluxes of He-like triplets from $\mathrm{O}$ vII in Table 1.

\section{ANALYSIS AND RESULTS \\ 3.1. Density Measurement}

The He-like transitions, consisting of the resonance line (r) $1 \mathrm{~s}^{2}{ }^{1} \mathrm{~S}_{0}-1 \mathrm{~s} 2 \mathrm{p}{ }^{1} \mathrm{p}_{1}$, the intercombination line (i) 
TABLE 1

OBSERVED O VII LINE FLUXES DURING THE $\mathrm{Q}$ AND $\mathrm{F} 2+\mathrm{U}$ STATES

\begin{tabular}{cccc}
\hline \hline \multicolumn{2}{c}{ Quiescent (Q) } & \multicolumn{2}{c}{ Flaring } \\
$\lambda(\stackrel{\mathrm{O}}{A})$ & Flux $^{a}$ & $\lambda(\AA)$ & Flux $^{a}$ \\
\hline $22.08(\mathrm{f})$ & $2.1 \pm 0.2$ & $22.08(\mathrm{f})$ & $2.2 \pm 0.3$ \\
$21.78(\mathrm{i})$ & $0.8 \pm 0.2$ & $21.77(\mathrm{i})$ & $0.9 \pm 0.2$ \\
$21.58(\mathrm{r})$ & $3.0 \pm 0.3$ & $21.57(\mathrm{r})$ & $3.4 \pm 0.3$ \\
\hline
\end{tabular}

${ }^{a}$ Measured flux in $10^{-4}$ photons $/ \mathrm{cm}^{2} / \mathrm{s}$

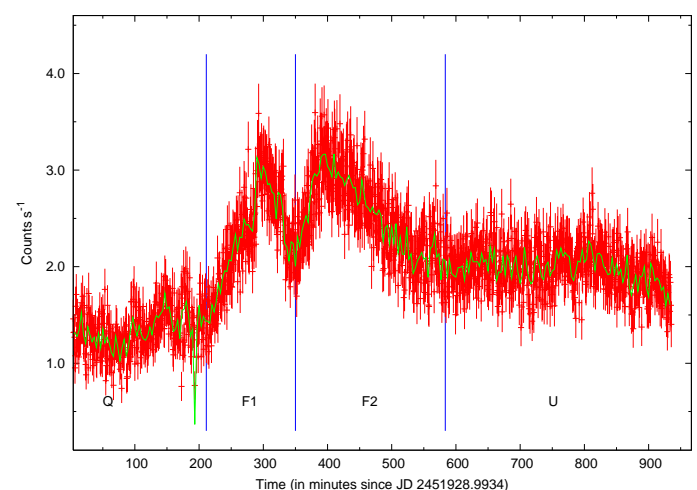

FIG. 1. - The X-ray 40-s binned light curve of the star $\xi$ Boo in the energy band $0.3-10$. $\mathrm{keV}$. The overplotted continuous line is $200 \mathrm{~s}$ binned light curve

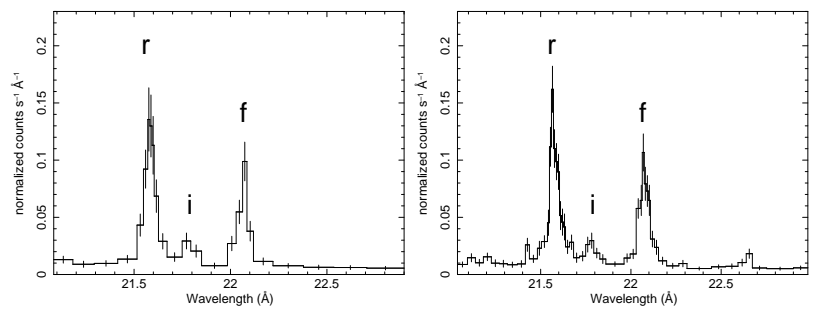

FIG. 2.- The O VII line of $\xi$ Boo observed by RGS1. Left panel: Quiescent state 'Q' and right panel: Flaring state 'F2+U'.

$1 \mathrm{~s}^{2}{ }^{1} \mathrm{~S}_{0}-1 \mathrm{~s} .2 \mathrm{p}{ }^{3} \mathrm{P} 1$, and the forbidden line (f) $1 \mathrm{~s}^{2}{ }^{1} \mathrm{~S}_{0}$ - $1 \mathrm{~s} .2 \mathrm{~s}{ }^{3} \mathrm{~S}_{1}$ are density- and temperature-dependent (e.g. Gabriel \& Jordan 1969). The intensity ratio $\mathrm{G}=(\mathrm{i}+\mathrm{f}) / \mathrm{r}$ varies with temperature and the ratio $R=f / i$ varies with electron density due to collisional coupling between the metastable $2^{3} \mathrm{~S}$ upper level of forbidden line and the $2^{3} \mathrm{P}$ upper level of the intercombination line. In the RGS wavelength, the $\mathrm{O}$ VII lines are clean, resolved and potentially suited to diagnose electron density and temperature (see Fig. 2). These lines at $21.60 \AA(\mathrm{r}), 21.80 \AA(\mathrm{i})$ and $22.10 \AA(\mathrm{f})$ have been used to obtain the temperature and density values from the $\mathrm{G}$ - and R-ratio using CHIANTI database (version 5.2.1; Landi et al. 2006). The G-ratio for $\xi$ Boo implies a temperature of $1.8 \pm 0.6$ and $2.0 \pm 0.6 \mathrm{MK}$ during the quiescent and flare states, respectively. For these temperatures, we derive electron densities of $n_{e}=1.3_{-0.5}^{+1.2} \times 10^{10} \mathrm{~cm}^{-3}$ for the quiescent state $(\mathrm{Q})$ and $n_{o}=1.6_{-0.8}^{+1.2} \times 10^{10} \mathrm{~cm}^{-3}$ for flare state $(\mathrm{F} 2+\mathrm{U})$. The electron density was slightly higher during the flare state. However, this increase is well within the $1 \sigma$ level.

\subsection{Loop Length}

The length of the stellar loops associated with the observed flaring activity can be estimated from the various loop models. Below we derive loop length from four different models.

\subsubsection{Hydrodynamic Flare model}

The details of this method are given in Pandey \& Singh (2008). The derived loop length for the flare F2 is found to be of $7.9 \pm 6 \times 10^{10} \mathrm{~cm}$. However, the value of $\zeta(=2$; slope of density temperature diagram) is outside the domain of the validity of the method. Therefore, the estimated loop length from this method may not be actual. Alternatively, Reale (2007) derived the loop length $(L)$ from the rise phase and peak phase of the flare. The loop length of the flare F2 from this approach is estimated to be $3.6 \pm 0.8 \times 10^{10} \mathrm{~cm}$.

\subsubsection{Pressure Balance method}

Shibata \& Yokoyama (2002) assume that the gas pressure is balanced by the magnetic pressure and give the equation for the magnetic field $(B)$ and the loop length $(L)$ :

$$
\begin{aligned}
& B=50\left(\frac{E M}{10^{48} \mathrm{~cm}^{-3}}\right)^{-1 / 5}\left(\frac{n_{e}}{10^{9} \mathrm{~cm}-3}\right)^{3 / 10}\left(\frac{T}{10^{7} \mathrm{~K}}\right)^{17 / 10} \\
& L=10^{9}\left(\frac{E M}{10^{48} \mathrm{~cm}^{-3}}\right)^{3 / 5}\left(\frac{n_{e}}{10^{9} \mathrm{~cm}^{-3}}\right)^{-2 / 5}\left(\frac{T}{10^{7} \mathrm{~K}}\right)^{-8 / 5}
\end{aligned}
$$

where $n_{e}$ is the density outside the flaring loop. By applying the average values of EM and T during the flare, we obtained a magnetic field strength of $29.4 \pm 6.0 \mathrm{G}$ and a loop length of $3.6 \pm 0.9 \times 10^{10} \mathrm{~cm}$ for the flare F2.

\subsubsection{Rise and decay time method}

The approach is described in Hawley et al. (1995). In this approach, the loop length has been derived by solving the time-dependent energy equation in rise and decay phase separately in different limits: strong evaporation driven by heating in the rise phase and strong condensation driven by radiation in the decay phase. The time rate change of the loop pressure $(\dot{p})$ for the spatial average is given by

$$
\frac{3}{2} \dot{p}=Q-R
$$

where $Q$ is the volumetric flaring heating rate, and $R$ is the optically thin cooling rate. The rise phase is dominated by strong evaporative heating, i.e., $Q>>R$, while the decay phase is dominated by cooling and strong condensation, i.e., $R>>Q$. At the loop top, there is an equilibrium, i.e., $Q=R$ and the loop length can be given as

$$
L=\frac{1500}{\left(1-x_{d}^{1.58}\right)^{4 / 7}} \cdot \tau_{d}^{4 / 7} \cdot \tau_{r}^{3 / 7} \cdot T_{A}^{1 / 2}
$$

where $\tau_{d}$ is the decay time, $\tau_{r}$ is the rise time, $T_{A}$ is the temperature at flare apex, and $x_{d}=c_{q} / c_{\max } . c_{\max }$ $(=3.16$ counts $/ \mathrm{s})$ and $c_{q}(=1.24$ counts $/ \mathrm{s})$ are count rate at the peak of the flare and the count rate during the quiescent state, respectively. Using this approach, the loop length for the flare F2 is estimated to be $3.9 \pm 0.5 \times$ $10^{10} \mathrm{~cm}$. The values of $\tau_{d}(=9885 \mathrm{~s}), \tau_{r}(=3351 \mathrm{~s})$, and $T_{A}(=13.3 \mathrm{MK})$ are taken from Pandey \& Singh (2008). 


\subsubsection{Haisch's Approach}

Given an estimate of two measured quantities, the emission measure (EM) and the decay time scale of the flare $\left(\tau_{d}\right)$ Haisch's approach (Haisch 1983) leads to the following expression for the loop length $(L)$

$$
L=5 \times 10^{-6} E M^{1 / 4} \tau_{d}^{3 / 4}
$$

Using this approach, we obtained a loop length of $3.8 \pm$ $0.2 \times 10^{10} \mathrm{~cm}$. The minimum magnetic field strength is estimated from the condition $B^{2} / 8 \pi \leq 2 n_{o} k_{B} T$, where $k_{B}$ is Boltzmann's constant. The minimum magnetic field strength thus obtained was 36 Gauss. This lies in the upper limit of the magnetic field determined by the pressure balance method.

The loop length derived from the above all four methods is consistent with each other.

\subsection{Wavelet and Periodogram Analyses}

We have used the wavelet analysis IDL code "Randomlet" developed by E. O'Shea. The program executes a randomization test (Linnell Nemec \& Nemec 1985; O'Shea et al. 2001) which is an additional feature along with the standard wavelet analysis code (Torrence \& Compo 1998) to examine the existence of statistically significant real periodicities in the time series data. The advantage of using this test is that it is distribution free or non parametric, i.e., it is not constrained by any specific noise model such as Poisson, Gaussian, etc. Using this technique, many important results have been published by analyzing approximately evenly sampled data (e.g., Banerjee et al. 2001; O'Shea et al. 2001, 2007; Srivastava et al. 2008a,b; O'Shea \& Doyle 2009; Gupta et al. 2009).

The wavelet power transforms of the U part of the Xray light curve of cadence $40 \mathrm{~s}$ (see the top-left panel of Fig. 3a) are shown in the middle left panel of Fig. 3a, where the darkest regions show the most enhanced oscillatory power in the intensity wavelet spectrum. The cross-hatched areas are the cone of influence (COI), the region of the power spectrum where edge effects, due to the finite lengths of the time series, are likely to dominate. The maximum allowed period from COI, where the edge effect is more effective, is $\sim 7363 \mathrm{~s}$. In our wavelet analysis, we only consider the power peaks and corresponding real periods (i.e. probability $>95 \%$ ) below this threshold. The period with maximum power detectable outside the COI is $\sim 2883 \mathrm{~s}$ with a probability of $\sim 94 \%$ and the repetition of only $\sim 3$ cycles in the time series. However, another peak is visible at a period of $\sim 1019$ $\mathrm{s}$ with a probability of $\sim 99 \%$ and the repetition of $\sim 11$ cycles. Therefore, only the periodicity of $\sim 1019$ s satisfies the recently reported more strict criteria of O'Shea \& Doyle (2009), i.e. at least four cycle of repetition over the life time of the oscillations. The middle-right panel in Fig. 3a shows the global wavelet power spectra of the time series from which the statistically significant period ( 1019s) is selected. In Fig. 3a, the bottom panel shows the probabilities of the presence of two specific frequencies (or periods) corresponding to the first and second highest peaks shown in the middle-left panel as functions of the time after the start of the observations. The solid line represents the probability corresponding to the
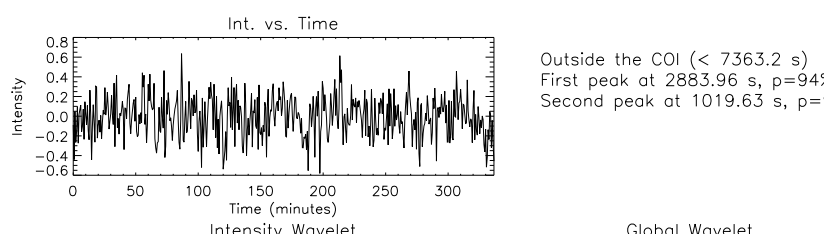
First peak at $2883.96 \mathrm{~s}, \mathrm{p}=94 \%$
Second peak at $1019.63 \mathrm{~s}, \mathrm{p}=99 \%$
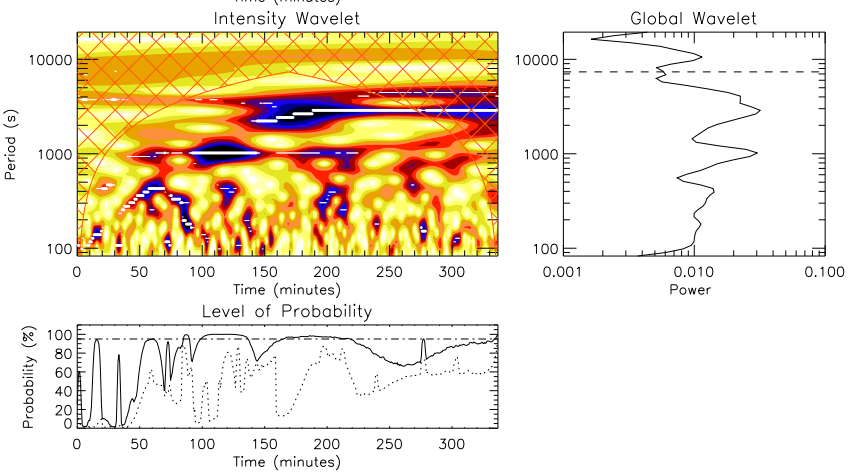

(a)

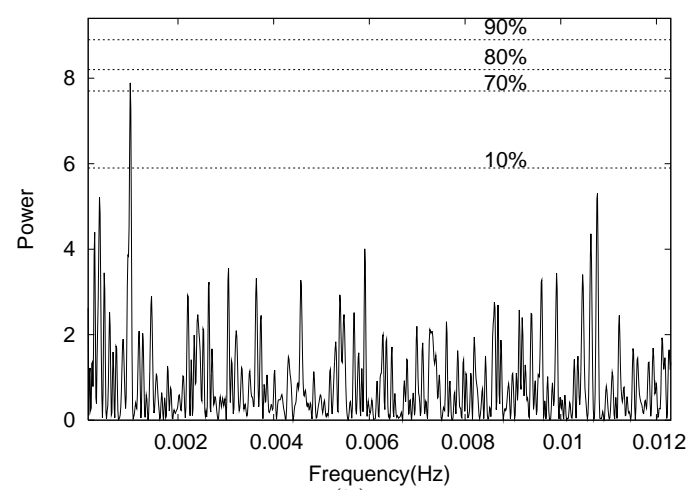

(b)

FIG. 3.- (a) The wavelet result for U part of the X-ray light curve of $\xi$ Boo. The top panel shows the variation of intensity, the wavelet power spectrum is given in the middle panel, and the probability in the bottom panel. (b) Power spectrum from periodogram analysis.

major power peak of time series data, while the dotted line corresponds to the secondary power peak. Wavelet analysis was also performed in the decay phase of the flare F2, and no significant periodicity was found.

We have also performed the periodogram analysis (Scargle 1982) in the U part of the X-ray light curve (see Fig. 3b). In the power spectrum, the highest peak corresponds to a period of $\sim 1005 \mathrm{~s}$ (probability $\sim 75 \%$ ), which is consistent with that obtained from wavelet analysis. Therefore, only the period of $\sim 1019 \mathrm{~s}$ appears to be statistically significant in our analyses.

\subsection{Fast-kink Oscillations}

The observed periodicity may be the likely signature of MHD oscillations associated with the stellar flare loops. The overdense magnetic loops are pressure balanced structures and may contain fast-kink and sausage oscillation modes with phase speed greater than the local Alfvénic speed $\left(\omega / k>v_{A}\right)$, and slow acoustic modes with sub-Alfvénic speed $\left(c_{0}<v_{A}\right)$ under coronal conditions.

The expressions for the oscillation periods of slow $\left(P_{s}\right)$, fast-kink $\left(P_{f k}\right)$, and fast-sausage $\left(P_{f s}\right)$ modes are given 
DERIVED PARAMETERS

TABLE 2

\begin{tabular}{|c|c|c|c|c|c|}
\hline \multirow{2}{*}{ Model } & \multirow{2}{*}{$\begin{array}{l}\text { Loop Length } \\
\left(10^{10}\right) \mathrm{cm}\end{array}$} & \multicolumn{3}{|c|}{ Theoretically estimated Period } & \multirow{2}{*}{$\begin{array}{c}\text { Observationally } \\
\text { Estimated } \\
\text { period (s) }\end{array}$} \\
\hline & & $\begin{array}{l}\text { Slow Mode } \\
(\mathrm{s})\end{array}$ & $\begin{array}{l}\text { Fast-Kink mode }_{(\mathrm{s})}^{a b} \\
\end{array}$ & $\begin{array}{c}\text { Fast Sausage }_{(\mathrm{s})} \\
\text { Mode }\end{array}$ & \\
\hline Hydrodynamic & $3.6 \pm 0.8$ & $1586 \pm 353$ & $1004 \pm 391$ & $313 \pm 121$ & \multirow{4}{*}{1019} \\
\hline Rise and decay & $3.9 \pm 0.5$ & $1718 \pm 222$ & $1087 \pm 374$ & $339 \pm 116$ & \\
\hline Pressure balance & $3.6 \pm 0.9$ & $1586 \pm 377$ & $1004 \pm 407$ & $313 \pm 127$ & \\
\hline Haisch's approach & $3.8 \pm 0.2$ & $1674 \pm 92$ & $1059 \pm 343$ & $330 \pm 107$ & \\
\hline
\end{tabular}

as follows (Edwin \& Roberts, 1983; Roberts et al., 1984; Aschwanden et al., 1999) :

$$
\begin{gathered}
P_{s}=\frac{2 L}{j c_{T}}=\frac{2 L}{j c_{o}}\left[1+\left(\frac{c_{o}}{v_{A}}\right)^{2}\right]^{1 / 2} \approx 1300 \frac{L_{10}}{\sqrt{T}_{6}} s \\
P_{f k}=\frac{2 L}{j c_{k}}=4 \pi^{1 / 2} \frac{L}{j}\left(\frac{\rho_{o}+\rho_{e}}{B_{o}^{2}+B_{e}^{2}}\right)^{1 / 2} \approx 205 \frac{L_{10} \sqrt{n_{9}}}{B_{10}} s \\
P_{f s}=\frac{2 \pi a}{c_{k}}=4 \pi^{3 / 2} a\left(\frac{\rho_{o}+\rho_{e}}{B_{o}^{2}+B_{e}^{2}}\right)^{1 / 2} \approx 6.4 \frac{a_{8} \sqrt{n_{9}}}{B_{10}} s
\end{gathered}
$$

where $L, a, \rho$, and $B$ are the loop length, the loop width, the ion mass density and magnetic field, respectively. The $c_{T}=c_{o} v_{A} /\left(c_{o}^{2}+v_{A}^{2}\right)^{1 / 2}$, where $c_{O}$ and $v_{A}$ are the sound and Alfvénic speeds respectively. The $c_{k}$ is the phase speed. Under coronal conditions, $B_{o} \approx B_{e}$, $\rho_{e}<<\rho_{o}, n_{H} \approx n_{e}$, and $c_{o}<<v_{A}$. The subscripts ' $o^{\prime}$ and ' $e$ ' stands for 'inside', and 'outside' of the loop. The $j$ is the number of nodes in fast-kink and slow oscillations, and is equal to 1 for the fundamental mode. The approximate relation in Equations 6-8 are given in coronal conditions for the fundamental oscillation periods of different modes in magnetic loops. Therefore, these approximate equations are also valid for the stellar loops in the coronae of magnetically active Sun-like stars. The parameters are expressed in units of $L_{10}=L / 10^{10} \mathrm{~cm}$, $a_{8}=a / 10^{8} \mathrm{~cm}, n_{9}=n_{o} / 10^{9} \mathrm{~cm}^{-3}, T=T_{e} / 10^{6} \mathrm{~K}$, and $B_{10}=B / 10 G$. The loop width was determined by assuming $a=0.1 L$ (Shimizu 1995) for a single loop.

Using the observationally estimated loop length and width, density, magnetic field, and Equations (6)-(8), the estimated periods for different oscillation modes are given in Table 2. The observationally derived oscillation period $\sim 1019 \mathrm{~s}$ approximately matches with the theoretically derived oscillation period for a fundamental fast kink mode.

\section{DISCUSSION AND CONCLUSIONS}

We found the first signature of fundamental kink oscillations with period of $\sim 1019 \mathrm{~s}$ in the magnetically active star $\xi$ Boo observed by EPIC-MOS of XMM-Newton, which may be formed either by the superposition of oppositely propagating fast kink waves or due to flare generated disturbances near the loop apex. The flaring activity may be the possible mechanism of the generation of such oscillations in the stellar loops. Recently, Cooper et al. (2003) have found that the transverse kink modes of fast magneto-acoustic waves may modulate the emission observable with imaging telescopes despite their incompressible nature. This is possible only when the axis of an oscillating loop is not orientated perpendicular to the observer's line of sight.

Resonant absorption is the most efficient mechanism theorized for the damping of a kink mode in which energy of the mode is transferred to the localized Alfvénic oscillations of the inhomogeneous layers at the loop boundary (Lee \& Roberts 1986). Intensity oscillations due to the fast-kink mode of magneto-acoustic waves do not show any decay of the amplitude during the initial 250 minutes in the post-flare phase observations of $\xi$ Boo. This is probably due to an impulsive driver, which forces such kind of oscillations and dominates over the dissipative processes during the flare $\mathrm{F} 2$ of $\xi$ Boo.

In conclusion, we report the first observational evidence of fundamental fast-kink oscillations in stellar loops during a post-flare phase of heightened emission in the cool active star $\xi$ Boo. These oscillations are a unique tool for the estimation of several key parameters of coronae, which are not directly measurable, e.g., the magnetic field. Some of the seismological techniques developed for the solar corona, e.g., based upon the ratio of the periods of kink modes, do not require the spatial resolution, and hence can be applied to estimate the density scale heights of the stellar coronae. Therefore, based on the solar analogy, such observations may shed new lights on the dynamics of the magnetically structured stellar coronae and its local plasma conditions. However, a future multiwavelength observational search is required to investigate various kinds of MHD modes to probe the local conditions of plasma in magnetically active stars.

We thank referee for his valuable comments and suggestions. We thank E. O'Shea for 'Randomlet'. We also thank M. J. Aschwanden, V. M. Nakariakov, L. Ofman, R. Sagar, and T. V. Zaqarashvili for carefully reading the manuscript and valuable suggestions. The research is based on observations obtained with XMM-Newton, an ESA science mission with instruments and contributions directly funded by ESA Member States and NASA.

\section{REFERENCES}

Aschwanden, M. J., Fletcher, L., Schrijver, C. J., \& Alexander, D. 1999, ApJ, 520, 880

Banerjee, D., O'Shea, E., Doyle, J. G., \& Goossens, M. 2001, A\&A, 377, 691

Cooper, F. C., Nakariakov, V. M., \& Tsiklauri, D. 2003, A\&A, 397,765
Edwin, P. M., \& Roberts, B. 1983, Sol. Phys., 88, 179

Gabriel, A. H., \& Jordan, C. 1969, Nature, 221, 947

Gupta, A. C., Srivastava, A. K., \& Wiita, P. J. 2009, ApJ, 690, 216

Haisch, B. M. 1983, in Proc. IAU Colloq. 71, Activity in Red-Dwarf Stars, Vol. 102 (Dordrecht: Reidel), 255 
Hawley, S. L., et al. 1995, ApJ, 453, 464

Jansen, F., et al. 2001, A\&A, 365, L1

Laming, J. M., \& Drake, J. J. 1999, ApJ, 516, 324

Landi, E., Del Zanna, G., Young, P. R., Dere, K. P., Mason, H. E., \& Landini, M. 2006, ApJS, 162, 261

Lee, M. A., \& Roberts, B. 1986, ApJ, 301, 430

Linnell Nemec, A. F., \& Nemec, J. M. 1985, AJ, 90, 2317

Mathioudakis, M., Bloomfield, D. S., Jess, D. B., Dhillon, V. S., \& Marsh, T. R. 2006, A\&A, 456, 323

Mitra-Kraev, U., Harra, L. K., Williams, D. R., \& Kraev, E. 2005, A\&A, 436, 1041

Mullan, D. J., Herr, R. B., \& Bhattacharyya, S. 1992, ApJ, 391, 265

Nakariakov, V. M., \& Verwichte, E. 2005, Living Reviews in Solar Physics, 2, 3

Nakariakov, V. M. 2007, Advances in Space Research, 39, 1804

O'Shea, E., \& Doyle, J. G. 2009, A\&A, 494, 355

O'Shea, E., Srivastava, A. K., Doyle, J. G., \& Banerjee, D. 2007, A\&A, 473, L13

O'Shea, E., Banerjee, D., Doyle, J. G., Fleck, B., \& Murtagh, F. 2001, A\&A, 368, 1095
Pandey, J. C., \& Singh, K. P. 2008, MNRAS, 387, 1627

Reale, F. 2007, A\&A, 471, 271

Roberts, B., Edwin, P. M., \& Benz, A. O. 1984, ApJ, 279, 857

Scargle, J. D. 1982, ApJ, 263, 835

Shibata, K., \& Yokoyama, T. 2002, ApJ, 577, 422

Smith, R. K., Brickhouse, N. S., Liedahl, D. A., \& Raymond, J. C. 2001, ApJ, 556, L91

Shimizu T., 1995, PASJ, 47, 251

Srivastava, A. K., Zaqarashvili, T. V., Uddin, W., Dwivedi, B. N., \& Kumar, P. 2008a, MNRAS, 388, 1899

Srivastava, A. K., Kuridze, D., Zaqarashvili, T. V., \& Dwivedi, B. N. 2008b, A\&A, 481, L95

Torrence, C., \& Compo, G. P. 1998, Bulletin of the American Meteorological Society, 79, 61

Van Doorsselaere T., Nakariakov V. M., Verwichte E., 2008, ApJ, 676, L73 